\title{
Variance Value Limited Clipping of Pentile based Sub-histogram Equalization for Contrast Enhancement of Image
}

\author{
Kuldip Acharya \\ Department of Computer Science and Engineering, National Institute of Technology, Agartala, Barjala, Jirania, Tripura \\ (West), Pin: 799046, India \\ Email: kuldip.acharjee@gmail.com

\section{Dibyendu Ghoshal} \\ Department of Electronics and Communication Engineering, National Institute of Technology, Agartala, Barjala, Jirania, \\ Tripura (West), Pin: 799046, India \\ Email: tukumw@gmail.com
}

Received: 17 February 2020; Accepted: 02 July 2020; Published: 08 December 2020

\begin{abstract}
Digital image enhancement is a technique to process a digital image to improve the overall visual quality of image. In this paper, Variance concept based clipping threshold value is computed from input image pixel intensity to limit the rate of over enhancement. The histogram of the original image is sub-divided into five adjacent sections and the boundary values between adjacent sections are put from the penile value of intensity range. Besides, over enhancement of the image is avoided by clipping certain number of pixels having more intensity than the clipping limit and these pixels are rearranged below the clipping limit. The present method offers two advantages viz., clipping of the certain pixels based on the property of the data set itself. The another one is to histogram processing by parts and this has given better visual quality, low computation time with improved metrics related to image enhancement. Histogram of each specific sub-image is equalized independently and then combined to produce the final contrast enhanced image. The final output image is further processed through imreducehaze filter for more improve result. Quantitative evaluation of proposed algorithm is performed by CPCQI and QILV image quality metrics and the simulation results have shown that the proposed variance based histogram equalization algorithm produces better quality of image in terms of contrasts, brightness and color in comparison to the other existing histogram equalization algorithms.
\end{abstract}

Index Terms: Contrast enhancement, Histogram clipping, Histogram Equalization, Image enhancement, Variance.

\section{Introduction}

Image enhancement is an important branch in digital image processing to identify the key features [1] of images by removing the noise, adjusting the contrast, color and brightness of images for further image analysis. Image enhancement improves the visual quality and appearance of digital images for the human visualization [2]. The primary goal of the image enhancement is obtaining the attributes of an image to improve the visual quality. In histogram equalization processing phase one or more image properties are changed by different techniques. The image enhancement algorithm can be divided into the frequency domain and spatial domain, respectively [3]. Image pixels are directly manipulated in spatial domain to accomplished the desired enhancement process. In the frequency domain enhancement techniques, the image is initially transferred into frequency domain. The histogram equalization [4] is a conventional process for contrast enhancement of digital image and a numerous study associated to this area have done for a better image enhancement viz. conventional histogram equalization (HE) [5], Bi-Histogram Equalization (BBHE) [6], RMSHE [5] is an improvement of BBHE. RMSHE method separate the input image histogram recursively on the computed mean value of image. The entire image is partitioned in many sub parts in the local histogram equalization technique and each part is processed separately. Lastly, the processed sub-images are integrated to gain the final enhanced image. The digital image enhancement algorithm is used for improvement of the information in images for better human visualization and understanding. Chen et.al. introduce minimum mean brightness error bi-histogram equalization (MMBEBHE) image enhancement [7] algorithm for preserving the mean brightness. MMBEBHE method is an extension of BBHE algorithm, which calculates the absolute mean brightness error (AMBE) and separate the histogram into two part based on minimum AMBE value. Yu Wan et.al. proposed dualistic sub-image histogram 
equalization (DSIHE) [7] enhancement method which preserve the brightness and entropy of output image. Human visual system-based thresholding multi-histogram equalization [8] (HVSHE) method is proposed by Wharton et.al. where logarithmic approach is applied for image enhancement. Exposure based sub image histogram equalization (ESIHE) [9] proposed by Singh et al for the low light image enhancement where histogram partition is done by the exposure threshold value. Recursive ESIHE (RESIHE) [10] usage average image intensity value as the histogram separating point for low exposure image enhancement. In median-mean based sub-image clipped histogram equalization (MMSICHE) [11] image enhancement method, a histogram of the image is sectioned into four sub histograms with the help of mean and median of image histogram. Images produced by MMSICHE method shows some artifacts on the output images. Tuned tri-threshold fuzzy intensification operators (TTFIO) [12] method use a fuzzy membership function used to set the image pixels values of a specified channel to the variation among 0 and 1 for enhancement of image with improved color. Multiscale Retinex method is broadly used for image enhancement by constraints of Gaussian scales, offset and gain, and so forth. PSO algorithm with advanced Retinex algorithm is used to fine tune the constraints for multiscale Retinex with chromaticity preservation (MSRCP) [13]. Jobson et al. suggest Multiscale Retinex with color (MSRCR) method initiated on Land's Retinex [14]. MSRCR method is introduced by Y. Liu et al. where three fidelity rules and numerical methods is used to keep the standards to estimate the reliability of image enhancement. So far as the best available literature and resources available to the present author, R-ESHIE [10] can be considered as the best among the aforesaid algorithms but still in this method also, dark regions are produced over dark pixel area which degrades the overall image enhancement to some extent.

The motivation of the present study was to find out some new methodology for deep level local histogram processing and to find some algorithm which can be helpful for enhancing the low illumination level digital images. In one of the earlier reports, histogram-oriented method was used and corresponding ESIHE [9] algorithm produced images with good contrast but little blur effect was found to be present in the output images. MMSICHE [11] methodbased method was found to produce images with undesired artifacts. Apart from this method, another RESHIE [10] method yielded apparently fair results with generation of certain dark regions which degraded overall performance. To overcome all these aforesaid problems, proposed method has been introduced with improved related metrics. In this paper the idea of subdivision of image histogram into five adjacent sub-section can be considered as novel and introduction of variance value as histogram clipping limit is also found out to be effective for various type of images and specially in low illumination level images. This would in turn help in deep level local processing of the histogram. Refer to the simulation results have shown that the proposed variance histogram equalization algorithm produces a better quality of image in terms of contrasts, brightness and color. In earlier studies, nobody did the histogram equalization by dividing the histogram spectrum into five equal parts. The current methodology is not only a novel one, but also it is capable to restrict over enhancement as well as better enhancement of the contrast of the image. This is obvious from visual perception as well as in terms of various related metrics.

\section{Preliminary Study}

\section{A. Variance of Image Histogram}

The variation of the lighting condition is an important aspect of digital image processing [15] in context of brightness and contrast of an image. Histogram of an image may contain both high contrast and low contrast, and both high brightness and low brightness in some area of an image. Analysis of variance is to compare those pixels region where the contrast and brightness changes. Mean value of image histogram gives each pixel intensity of the image from 0 to 255 or 0 to L-1 intensity levels and variance is usually computed to find out how each pixel varies from the neighboring pixel or center pixel of image histogram. In the proposed method variation is used in computation of the histogram clipping threshold value. Variance can be used to classify different pixel variation of image histogram.

\section{Algorithm of the Proposed Method}

Step 1: Take the histogram of input color image.

Step 2: Histogram subdivision in five equal parts is done. At first separation of the histogram is done into two parts and then further division of these histogram into three parts is done.

Step 3: Histogram clipping threshold value is calculated using variance operation.

Step 4: Computation of total number of pixels in all five sub images is done to be used in PDF computation of sub images.

Step 5: Both the probability density function (PDF) and cumulative distribution function (CDF) of all five sub images is accomplished.

Step 6: Transfer function is done for histogram equalization of all five sub images and then integrated all these subparts into one single final image.

Step 7: The further enhancement of output image is done by imreducehaze filter which gives robust result with natural color. 


\section{A. Contributions of the proposed algorithm}

In the proposed algorithm, a clipping threshold value is computed such that only those pixels exist below the clipping limit are allowed to control the over enhancement rate. Performance evaluation of the proposed algorithm is calculated and verified over a number of images of the publicly available BSD dataset [16]. Comparative study is also stated through the other common HE based algorithms using image quality metrics such as QILV [17], and CPCQI [18].

\section{Proposed Methodology}

Total number of image pixel is calculated in equation (1) as

$$
T P=\sum_{p=0}^{L-1} G(\mathrm{p})
$$

Here, $G(p)$ is the image histogram and $L$ represent maximum intensity level of an image and it range from 0 to 255 . Mean value calculation is given in equation (2) as

$$
\mu=\frac{\sum_{p=0}^{L-1} p \times G(\mathrm{p})}{\sum_{p=0}^{L-1} G(\mathrm{p})}
$$

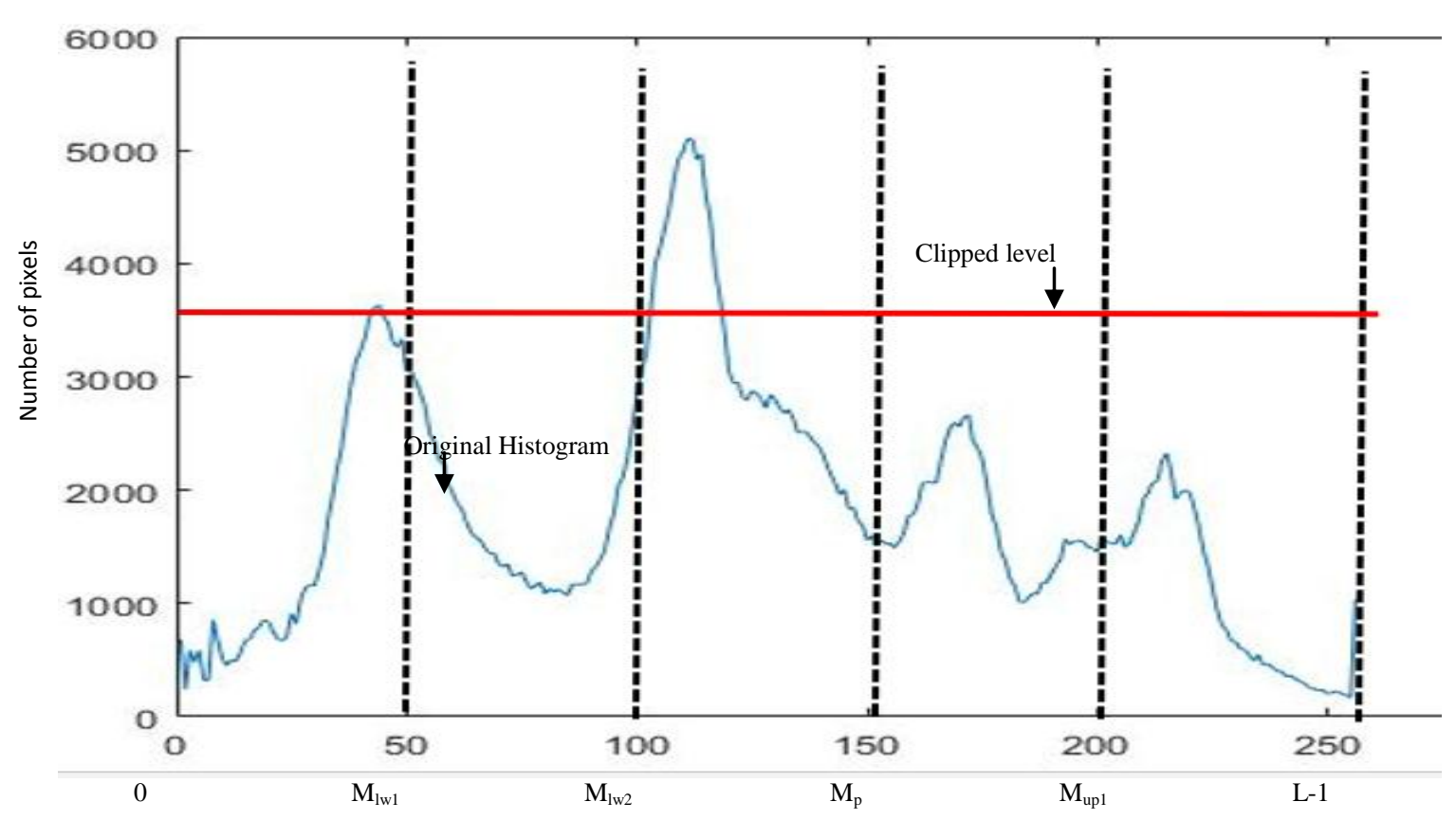

Fig.1 Histogram sub division in five parts and clipping process

Clipped histogram threshold value calculation using variance is given in equation (3)

$$
T h_{C l}=\frac{\sum_{p=0}^{L-1}(p-\mu)^{2} \times G(p)}{\sum_{p=0}^{L-1} G(p)}
$$

Clipped histogram equation is given in equation (4)

$$
G_{C l}(\mathrm{p})=\left\{\begin{array}{l}
T h_{C l} \text { if } G(\mathrm{p}) \geq \mathrm{Th}_{C l} \\
G(\mathrm{p}) \text { if } G(\mathrm{p})<T h_{C l}
\end{array}\right.
$$




\section{B. Histogram Sub Division Process}

$$
M_{p}=\frac{G(p)}{2}
$$

Lower limit and upper limit of image histogram is calculated in (6) and (7).

$$
\begin{gathered}
M_{l w}=M_{0}+M_{p} \\
M_{u p}=M_{L-1}-M_{p}
\end{gathered}
$$

$M_{l w}$ is lower limit of histogram and $M_{u p}$ is upper limit of the histogram is shown in Fig.1.

\section{Histogram Subdivision}

The original histogram is first divided into two part on an intensity value $\mathbf{M}_{\mathrm{p}}$ as computed in (5). These two separate sub-histograms are further separated into three small sub histograms and $\mathbf{M}_{\mathrm{lw}}$ and $\mathrm{M}_{\text {up }}$ are used as a histogram separation point of sub histograms as calculated in (4) and (5). Each five sub images. The pentile histogram subdivision process results in five sub images $\mathrm{SI}_{\mathrm{Ll}}, \mathrm{SI}_{\mathrm{L} 2}, \mathrm{SI}_{\mathrm{L} 3}, \mathrm{SI}_{\mathrm{Ul}}$ and $\mathrm{SI}_{\mathrm{Uu}}$ ranging from gray 0 to $\mathrm{M}_{\mathrm{lw} 1}, \mathrm{M}_{\mathrm{lw} 1}+1$ to $\mathrm{M}_{\mathrm{lw} 2}$, $\mathrm{M}_{\mathrm{lw} 2}+1$ to $\mathrm{M}_{\mathrm{p}}, \mathrm{M}_{\mathrm{p}}+1$ to $\mathrm{M}_{\mathrm{up} 1}, \mathrm{M}_{\mathrm{up} 1}+1$ to 255 shown in Fig.1. It shows the histogram sub division and clipping process through variance based the clipping process and pentile based histogram division, in five equal parts. $\mathrm{Pb}_{\mathrm{lw} 1}(\mathrm{p}), \mathrm{Pb}_{\mathrm{lw} 2}(\mathrm{p})$, $\mathrm{Pb}_{\mathrm{lw} 3}(\mathrm{p}), \mathrm{Pb}_{\mathrm{up} 1}(\mathrm{p})$, and $\mathrm{Pb}_{\mathrm{up} 2}(\mathrm{p})$ are computed PDF of these five sub images as expressed in the following equation (8)(12)

\section{Probability Density Function (PDF)}

$$
\begin{gathered}
P b_{l w 1}(p)=\frac{G_{C l}(p)}{T P_{l w 1}} \text { for } 0 \leq \mathrm{p} \leq \mathrm{M}_{l \mathrm{w} 1} \\
P b_{l w 2}(p)=\frac{G_{C l}(p)}{T P_{l w 2}} \text { for } \mathrm{M}_{l \mathrm{w} 1}+1 \leq \mathrm{p} \leq \mathrm{M}_{l \mathrm{w} 2} \\
P b_{l w 3}(p)=\frac{G_{C l}(p)}{T P_{l w 3}} \text { for } \mathrm{M}_{l \mathrm{w} 2}+1 \leq \mathrm{p} \leq \mathrm{M}_{\mathrm{p}} \\
P b_{u p 1}(p)=\frac{G_{C l}(p)}{T P_{u p 1}} \text { for } \mathrm{M}_{\mathrm{p}}+1 \leq \mathrm{p} \leq \mathrm{M}_{\mathrm{up} 1} \\
P b_{u p 2}(p)=\frac{G_{C l}(p)}{T P_{u p 2}} \text { for } \mathrm{M}_{\mathrm{up} 1}+1 \leq \mathrm{p} \leq \mathrm{L}-1
\end{gathered}
$$

The total number of pixels in each sub images are $\mathrm{TP}_{\mathrm{lw} 1}, \mathrm{TP}_{\mathrm{lw} 2}, \mathrm{TP}_{\mathrm{lw} 3}, \mathrm{TP}_{\mathrm{up} 1}$ and $\mathrm{TP}_{\mathrm{up} 2}$, respectively.

Cumulative density function (CDF) of each sub images are $\mathrm{Cd}_{\mathrm{lw} 1}(\mathrm{p}), \mathrm{Cd}_{\mathrm{lw} 2}(\mathrm{p}), \mathrm{Cd}_{\mathrm{lw} 3}(\mathrm{p}), \mathrm{Cd}_{\mathrm{up} 1}(\mathrm{p})$, and $\mathrm{Cd}_{\mathrm{up} 2}(\mathrm{p})$ are computed $\mathrm{CDF}$ of these five sub images as expressed in the following equation (13)-(17)

E. Cumulative Distribution Function

$$
\begin{gathered}
C d_{l w 1}(p)=\sum_{p=0}^{M_{l w 1}} P b_{l w 1}(p) \\
C d_{l w 2}(p)=\sum_{p=M_{l w 1}}^{M_{l w 2}} P b_{l w 2}(p) \\
C d_{l w 3}(p)=\sum_{p=M_{l w 2}+1}^{M_{p}} P b_{l w 3}(p)
\end{gathered}
$$




$$
\begin{gathered}
C d_{u p 1}(p)=\sum_{p=M_{p}+1}^{M_{u p 1}} P b_{u p 1}(p) \\
C d_{u p 2}(p)=\sum_{p=M_{p}+1}^{L-1} P b_{u p 2}(p)
\end{gathered}
$$

PDF and CDF are standard statistical parameters used to handle the random valued data and thus found to be pertinent to the present context of deep level local histogram processing.

F. Histogram Equalization by The Transfer Functions

$$
\begin{gathered}
F T_{1}=M_{l w 1} \times C d_{l w 1} \\
F T_{2}=\left(M_{l w 1}+1\right)+\left(\mathrm{M}_{l w 2}-M_{l w 1}+1\right) \times C d_{l w 2} \\
F T_{3}=\left(M_{l w 2}+1\right)+\left(\mathrm{M}_{p}-M_{l w 2}+1\right) \times C d_{l w 3} \\
F T_{4}=\left(M_{p}+1\right)+\left(\mathrm{M}_{u p 1}-M_{p}+1\right) \times C d_{u p 1} \\
F T_{5}=\left(M_{u p 1}+1\right)+\left(\mathrm{L}-M_{u p 1}+1\right) \times C d_{u p 2}
\end{gathered}
$$

The transfer functions $\mathrm{FT}_{1}, \mathrm{FT}_{2}, \mathrm{FT}_{3}, \mathrm{FT}_{4}$ and $\mathrm{FT}_{5}$ are used for equalizing the five sub histograms individually. Thereafter all the five sub images $\mathrm{S}_{\mathrm{lw} 1}, \mathrm{~S}_{\mathrm{lw} 2}, \mathrm{~S}_{\mathrm{lw} 3}, \mathrm{~S}_{\mathrm{up} 1}$ and $\mathrm{S}_{\mathrm{up} 2}$ are integrated into one complete image to form the output image.

This output image is further enhanced by using imreducehaze filter [17] which further improve the color quality of the image.

\section{Simulation Results and Analysis}

\section{A. Quality assessment of enhanced image}

Simulation in Matlab was achieved on 100 BSD (Berkeley Segmentation Database) images [16]. All the algorithms are run on a Windows 10 operating system with Intel core i7 processor and 8 GB random access memory. The algorithms are implemented in MATLAB [19] R2018a with the support of default constraints stated in the precise algorithms. Visual quality inspection and performance measurement of image by different algorithm, is implemented by the following image quality metrics, namely, QILV and C-PCQI.

B. Quality Index based on Local Variance (QILV) [18]

QILV between two images I and $\mathbf{J}$ is defined as

$$
Q I L V(I, J)=\frac{2 \mu V_{I} \mu V_{J}}{\mu^{2} V_{I}+\mu^{2} V_{J}} \times \frac{2 \sigma V_{I} \sigma V_{J}}{\sigma^{2} V_{I}+\sigma^{2} V_{J}} \times \frac{\sigma V_{I} V_{J}}{\sigma V_{I}+\sigma V_{J}}
$$

The covariance between the variances of two images $I$ and $J$ is expressed as

$$
\sigma V_{I} V_{J}=E\left\{\left(\operatorname{Var}\left(\mathrm{I}_{i, j}\right)-\mu V_{1}\right)\left(\operatorname{Var}\left(\mathrm{J}_{i, j}\right)-\mu V_{j}\right)\right.
$$

Here, the local variances of image $\mathrm{I}$ and $\mathrm{J}$ is denoted by $\mathrm{V}_{\mathrm{I}}$ and $\mathrm{V}_{\mathrm{J}}$, respectively. The computed mean values of the local variances of image I and $\mathrm{J}$, is represent by $\mu V_{1}$ and $\mu V_{j}$, respectively. The covariance between the variances of the two images is expressed by $\sigma V_{I} V_{J}$. The standard deviations of the local variance of image is given by $\sigma V_{I}$ and $\sigma V_{J}$. Both SSIM and QILV image quality metric return a scalar value between 0 and 1 and the values close to 1 signifies to high quality images. Table 1 . shows the average quality index based on local variance (QILV) in bold face. It is observed from the results that the proposed method gives the highest QILV for all images. Average QILV value of the 
proposed method is 0.9427 . Which demonstrates the best outcome with respect to the other algorithms. The second-best result for average QILV is given by RESIHE which returns average QILV value is 0.9364 .

\section{The Colorfulness based PCQI (C-PCQI) [19]}

The proposed PCQI metric is very correlated by subjective quality scores on image enhancement, but it does not consider the impact of colorfulness and this is a significant index of image quality assessment. The Colorfulness based PCQI (C-PCQI) metric is proposed to overcome this problem. The Colorfulness based PCQI (C-PCQI) metric is defined as

$$
S_{C P C Q I}=\frac{1}{N} \sum q_{m i}(i) \cdot \mathrm{q}_{c c}(i) \cdot \mathrm{q}_{s d}(i) \cdot \mathrm{q}_{c s}(i)
$$

$\mathrm{N}$ is the number of pixels. Here, $\mathrm{Q}_{\mathrm{mi}}$, signify the similarity through mean intensity between the original image and distorted image, $\mathrm{Q}_{\mathrm{cc}}$ denote the contrast alteration and $\mathrm{Q}_{\mathrm{sd}}$ represent the structural distortion between the original image and distorted image. Information regarding these three terms are reported in [35].

$$
Q_{c s}=\left(\frac{2 S T_{1} \cdot S T_{2}+\ell}{S T_{1}^{2} \cdot S T_{2}^{2}+\ell}\right) \delta
$$

The color saturation of the original and distorted images is denoted by $\mathrm{ST}_{1}$ and $\mathrm{ST}_{2}$, respectively. A very small constant number $\ell$ is used to avoid division-by-zero and a fixed pooling index denoted by $\delta$ used for stressing the location which have exceptional changes of image color saturation. Table 2 shows the C-PCQI results of different algorithms. It is found that the proposed method gives the highest C-PCQI values for all images. Average C-PCQI value of the proposed algorithm is 1.1243 . Which is the best result with respect to other compared algorithms? The secondbest result for average C-PCQI is given by RESIHE which gives average C-PCQI value is 1.1031.

Fig. 2 shows the outcome of proposed algorithm with and without using imreducehaze function processed on BSD image '361010' [16] of 'Polo Games' to gain more natural enhancement. It is also showing the histogram of both original image and proposed algorithm. It is clear from the results that proposed method applying Matlab [20] inbuilt imreducehaze function gives slightly better output image. The overall result of the proposed algorithm produces high quality image with natural vibrant color.

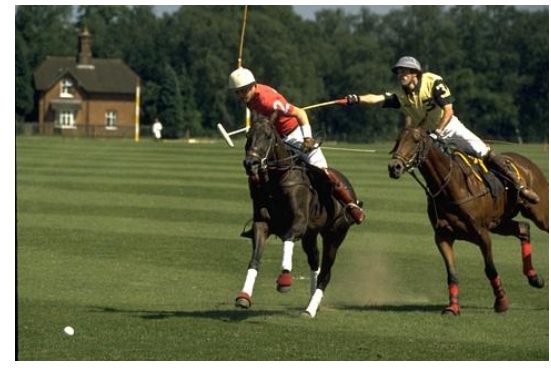

(a)

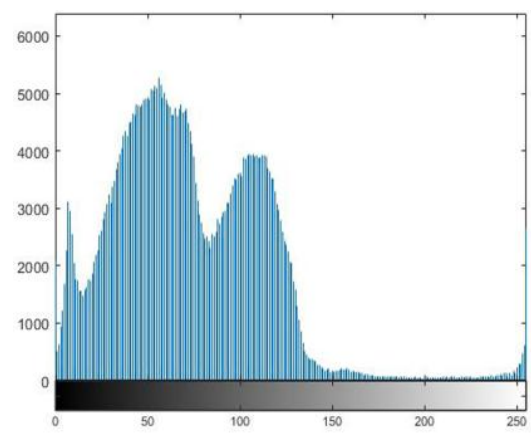

(d)

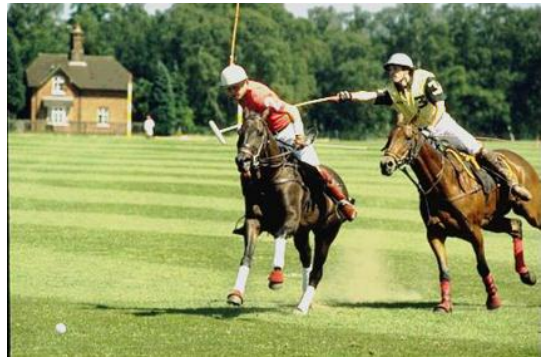

(b)

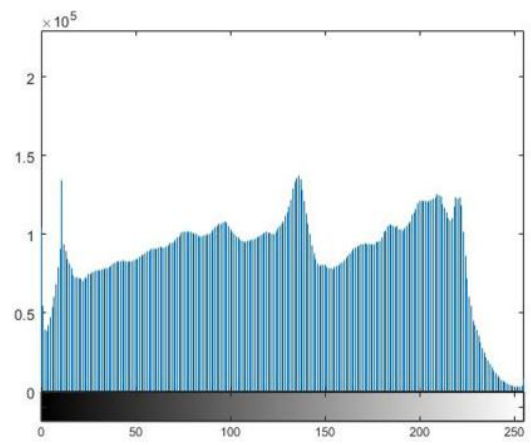

(e)

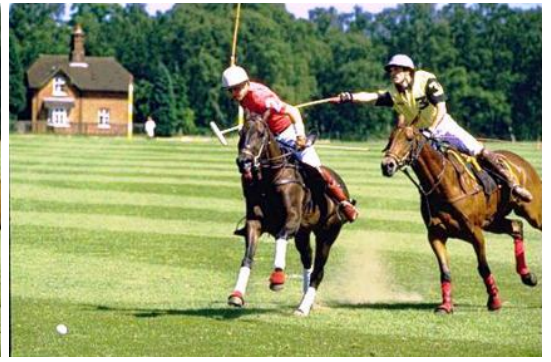

(c)

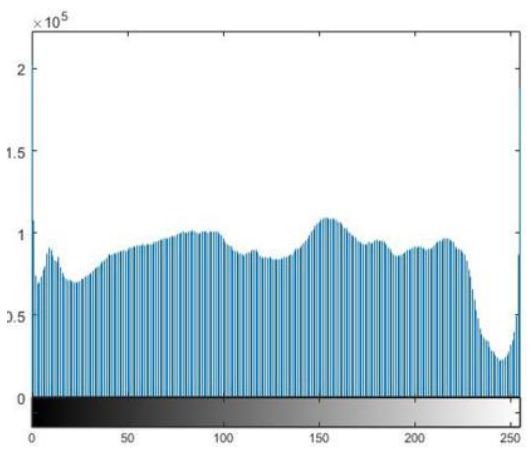

(f)

Fig. 2. (a) Original BSD image '361010' of 'Polo Games' (b) Proposed method without imreducehaze filter (c) Proposed method with imreducehaze filter (d) Original image histogram (e) Histogram of proposed method without imreducehaze filter (f) Histogram of proposed method with imreducehaze filter. 
Table 1. QILV results of different algorithms

\begin{tabular}{lllllll}
\hline BSD Image Number & ESIHE & MSRCR & MSRCP & TTFIO & RESIHE & Proposed \\
\hline "217090" & 0.93024 & 0.51925 & 0.32065 & $5.91 \mathrm{E}-11$ & 0.91479 & 0.9199 \\
"226033" & 0.95773 & 0.4395 & 0.57358 & $4.73 \mathrm{E}-11$ & 0.94196 & 0.9454 \\
"384022" & 0.97899 & 0.55839 & 0.33316 & $5.66 \mathrm{E}-12$ & 0.97418 & 0.9794 \\
"6046" & 0.98959 & 0.56458 & 0.5588 & $4.31 \mathrm{E}-12$ & 0.98209 & 0.9901 \\
"64061" & 0.81941 & 0.729 & 0.99998 & $2.24 \mathrm{E}-11$ & 0.81977 & 0.8251 \\
"71076" & 0.95141 & 0.56675 & 0.43427 & $1.23 \mathrm{E}-11$ & 0.93921 & 0.9528 \\
"207038" & 0.52782 & 0.64956 & $1.34 \mathrm{E}-11$ & 0.98966 & 0.98349 & 0.9868 \\
Average & $\mathbf{0 . 8 7 9 3}$ & $\mathbf{0 . 5 7 5 2}$ & $\mathbf{0 . 4 6 0 0}$ & $\mathbf{0 . 1 4 1 3}$ & $\mathbf{0 . 9 3 6 4}$ & $\mathbf{0 . 9 4 2 7}$ \\
\hline
\end{tabular}

Table 2. CPCQI results of different algorithms

\begin{tabular}{lllllll}
\hline BSD Image Number & ESIHE & MSRCR & MSRCP & TTFIO & RESIHE & Proposed \\
\hline 217090 & 1.0637 & 0.47958 & 0.51325 & 0.027874 & 1.0759 & 1.0866 \\
"226033" & 1.1457 & 0.48778 & 0.61882 & 0.033729 & 1.1563 & 1.1631 \\
"384022" & 1.0551 & 0.45046 & 0.63917 & 0.09247 & 1.0683 & 1.0958 \\
"6046" & 1.0456 & 0.42868 & 0.48105 & 0.059703 & 1.0545 & 1.1032 \\
"64061" & 1.1463 & 0.67313 & 0.80232 & 0.09209 & 1.1414 & 1.151 \\
"71076" & 1.0898 & 0.37032 & 0.50172 & 0.037589 & 1.1006 & 1.1232 \\
"207038" & 1.0647 & 0.72448 & 0.62872 & 0.038577 & 1.0975 & 1.1097 \\
Average & $\mathbf{1 . 0 9 1 2}$ & $\mathbf{0 . 5 2 2 4}$ & $\mathbf{0 . 6 1 1 9}$ & $\mathbf{0 . 0 5 9 0}$ & $\mathbf{1 . 1 0 3 1}$ & $\mathbf{1 . 1 2 4 3}$ \\
\hline
\end{tabular}

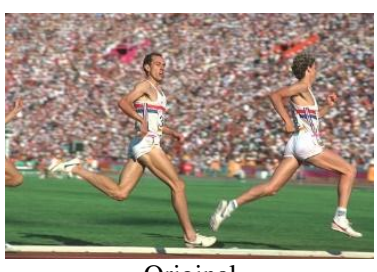

Original

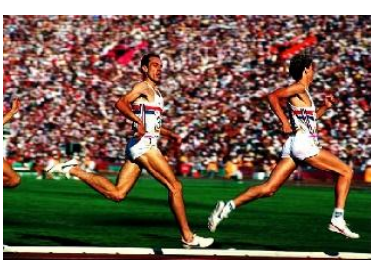

TTFIO

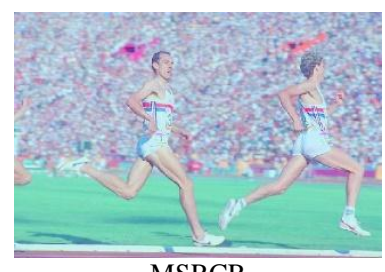

MSRCR

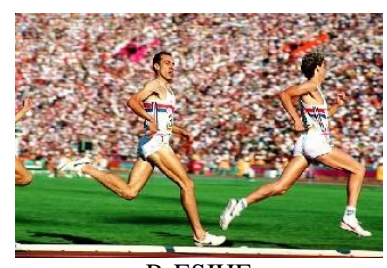

R-ESIHE

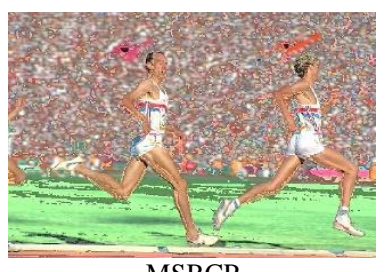

MSRCP

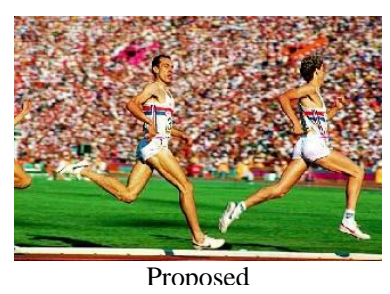

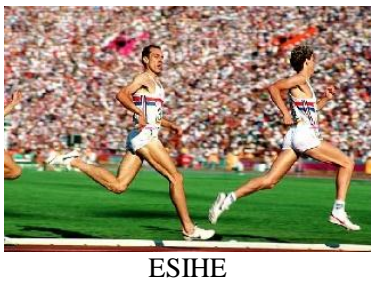

ESIHE

Fig.3. BSD Image '226033' of 'Sport Run'

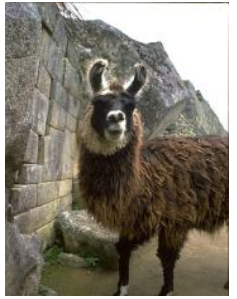

Original

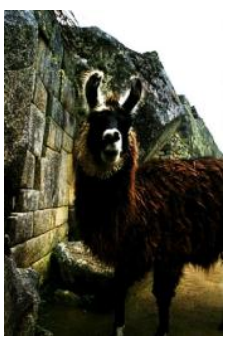

TTFIO

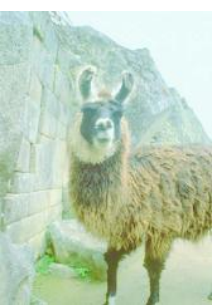

MSRCR

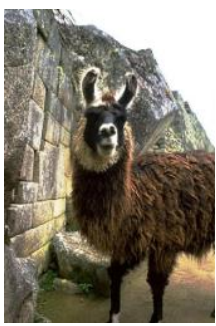

R-ESIHE

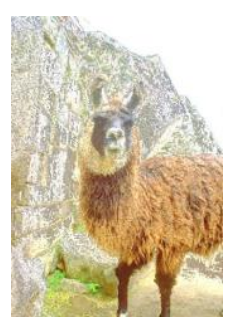

MSRCP

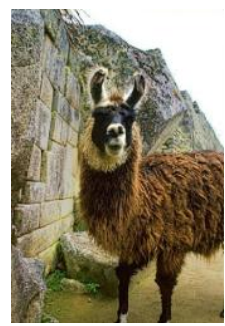

Proposed

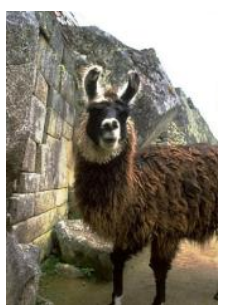

ESIHE

Fig.4. BSD Image 6046 of 'Mountain Goat' 
The BSD [16] image '226033' of 'Sport Run' and its enhancement results from different enhancement algorithm are shown in Fig.3. It is observed that MSRCR algorithm produces whitish, blurry and faded image. The overall visual appearance is unclear. MSRCP algorithm output image have noise, artifact and lost many details of object which causes poor visual quality. ESIHE algorithm produces good quality of image with less contrast. TTFIO algorithm output image is very dark which makes the object unclear to understand. R-ESIHE algorithm outperform than previous algorithms i.e., MSRCR, MSRCP, ESIHE, and TTFIO. But the pixels on the ground area is little darker. The proposed algorithm overcome from all these problems and it produces superior quality of image in comparison to other algorithm in context of color, brightness and contrast. The overall visual quality of proposed algorithm is the best.

The BSD image [16] '6046 of 'Mountain Goat' is shown in Fig.4. MSRCR algorithm generate image with faded, hazy, and blurry effects and this algorithm is disturbed by noise. The output image shows that 'Mountain goat' image have some artifact. MSRCP algorithm have lost many important information of image object. Over contrast and brightness degrades the image quality. ESIHE algorithm produced better result than MSRCR, MSRCP and TTFIO. But the image has slightly less contrast. TTFIO algorithm produces unclear image due to the dark region at some portion of the image. R-ESIHE algorithm produces better quality of image but the hairs of 'Mountain Goat' is slightly darker. The proposed algorithm overcomes from these problems. It produces best quality of image in terms of color, contrast and brightness compared to other algorithms. The visual performance of the proposed method is better than other enhancement algorithm.

The BSD image [16] '71076' of 'Statue of Man' is shown in Fig.5. The enhancement results of MSRCR algorithm produces blurred and washed-out image with less color. The overall visual presence is blurred. The output image obtain from MSRCP algorithm shows that the image color is disturbed, have noise, and artifact. It has lost many vital information of object and produces poor quality of image. The resultant image of ESIHE algorithm have less contrast and blur at the left middle side of wall. The output image given by TTFIO algorithm have some dark region which makes the object visibility unclear. R-ESIHE algorithm perform better than MSRCR, MSRCP, ESIHE, and TTFIO algorithm. The color and contrast of R-ESIHE algorithm is less compared to the proposed algorithm. The color, contrast and brightness of output image obtain from proposed algorithm is outstanding than all other compared algorithm. The overall visual quality of proposed algorithm is superior than others.

\section{Conclusion}

In this paper, a pentile division-based histogram equalization algorithm has been proposed for contrast enhancement of low illumination level images. It restricts the over enhancement of an image by histogram clipping process by a variance-based histogram clipping threshold value obtain from input histogram. The enhanced image is further processed through imreducehaze filtering for more natural enhancement. The algorithms are coded and run to evaluate various image quality metrics on Matlab platform and the results illustrate that the proposed algorithm gives superior quality of image than other histogram equalization-based algorithms. Both the quantitative and qualitative assessment results demonstrate the pre-eminence of the proposed algorithm in terms of natural image enhancement compared to other existing algorithms.

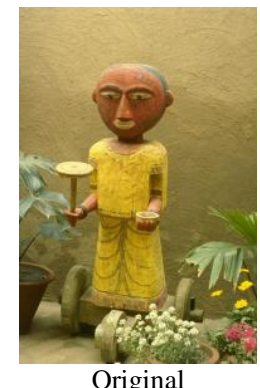

Original

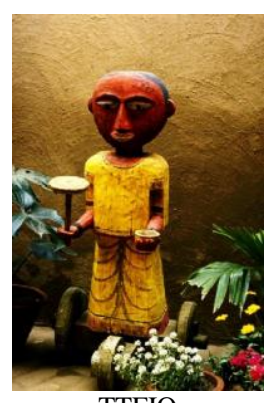

TTFIO

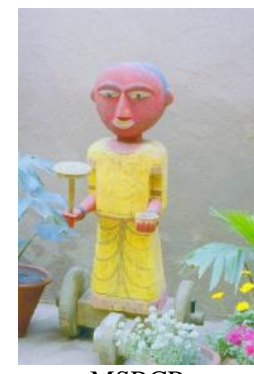

MSRCR

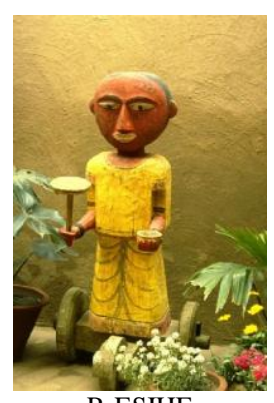

R-ESIHE

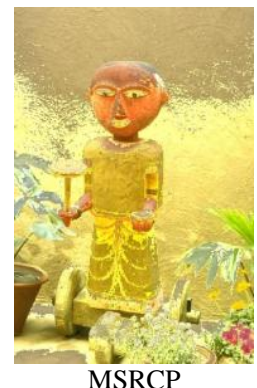

MSRCP

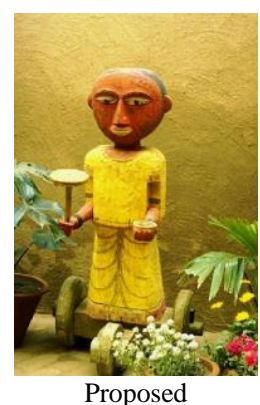

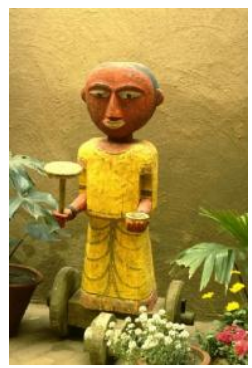

ESIHE

Fig.5. BSD Image 71076 of 'Statue of Man' 


\section{References}

[1] Enhancement methods in image processing, https://in.mathworks.com/discovery/image-enhancement.html, accessed February 12,2020

[2] H. R. Sheikh and A. C. Bovik, "Image information and visual quality," in IEEE Transactions on Image Processing, vol. 15, no. 2, pp. 430-444, Feb. 2006.

[3] R. C. Gonzalez, R. E. Woods, and S. L. Eddins, Digital Image Processing Using MATLAB. Prentice Hall, 2004.

[4] Kaur, Manpreet, Kaur, Jasdeep, and Kaur, Jappreet. Survey of contrast enhancement techniques based on histogram equalization. International Journal of Advanced Computer Science and Applications, 2(7):137-141, 2011.

[5] Soong-Der Chen, A. Rahman Ramli, "Preserving brightness in histogram equalization-based contrast enhancement techniques," Digital Signal Processing, 12(5), pp.413-428, September 2004.

[6] K. Singh, R. Kapoor, S. K. Sinha, "Enhancement of low exposure images via recursive histogram equalization algorithms", Optik-Int. J. Light Electron Opt., vol. 126, no. 20, pp. 2619-2625, 2015.

[7] Soong-Der Chen and A. R. Ramli, "Minimum mean brightness error bi-histogram equalization in contrast enhancement," in IEEE Transactions on Consumer Electronics, vol. 49, no. 4, pp. 1310-1319, Nov. 2003.

[8] E. Wharton, K. Panetta and S. Agaian, "Human Visual System Based Multi-Histogram Equalization for Non-Uniform Illumination and Shadow Correction," 2007 IEEE International Conference on Acoustics, Speech and Signal Processing ICASSP '07, Honolulu, HI, 2007, pp. I-729-I-732.

[9] K. Singh, R. Kapoor, "Image enhancement using exposure-based sub image histogram equalization", Pattern Recognition Letters, vol. 36, pp. 10-14, 2014.

[10] Singh, K., Kapoor, R., Sinha, S.K, 'Enhancement of low exposure images via recursive histogram equalization algorithms', Optik., 2015, 126, (20), pp. 2619-2625

[11] Singh, K., Kapoor, R, 'Image enhancement via median-mean based subimage-clipped histogram equalization', Optik., 2014, 125, (17), pp. 4646-4651

[12] Al-Ameen, Zohair, 'Visibility Enhancement for Images Captured in Dusty Weather via Tuned Tri-threshold Fuzzy Intensification Operators', International Journal of Intelligent Systems and Applications., 2016, 8, (8), pp. 10-17

[13] Matin, F., Jeong, Y., Kim, K., et al, 'Color image enhancement using multi scale retinex based on particle swarm optimization method', IOP Conf. Series: Journal of Physics: Conf. Series., 2018, 960, pp. 12026

[14] Jobson, D. J., Rahman, Z., Woodell, G. A, 'A multiscale retinex for bridging the gap between color images and the human observation of scenes', IEEE Transactions on Image Processing., 1997, 6, (7), pp. 965-976

[15] Haitao Wang, S. Z. Li and Yangsheng Wang, "Face recognition under varying lighting conditions using self quotient image," Sixth IEEE International Conference on Automatic Face and Gesture Recognition, 2004. Proceedings., Seoul, South Korea, 2004, pp. 819-824.

[16] Arbelaez, P., Maire, M., Fowlkes, C., et al, 'Contour Detection and Hierarchical Image Segmentation', IEEE Transactions on Pattern Analysis and Machine Intelligence., 2011, 33, (5), pp. 898-916

[17] Imreducehaze, https://in.mathworks.com/help/images/ref/imreducehaze.html, accessed February13, 2020

[18] S. Aja-Fernndez, R. San-Jos-Estpar, C. Alberola-Lpez, C.-F. Westin, "Image quality assessment based on local variance", Proc. 28th IEEE EMBC, pp. 4815-4818, 2006-Sep

[19] K. Gu, D. Tao, J.-F. Qiao, and W. Lin, "Learning a no-reference quality assessment model of enhanced images with big data," IEEE Trans. Neural Netw. Learn. Syst., to be published.

[20] MATLAB and Statistics Toolbox Release 2018a, The MathWorks, Inc., A Natick ed., Massachusetts, United States.

\section{Authors' Profiles}

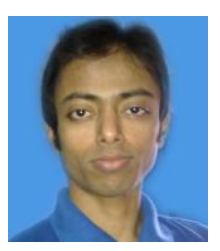

Kuldip Acharya received his M.Tech in Computer Science and Engineering from Tripura University, Tripura, India in 2012. He is doing Ph.D. in Computer Science \& Engineering from National Institute of Technology Agartala, India from 2013. His research areas of interest are image processing, computer vision, and 3D Computer Animation \& design.

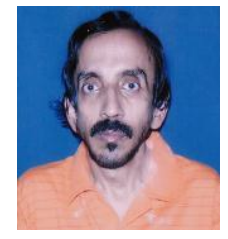

Dibyendu Ghoshal has received his B.Sc. (Honors in Physics), B.Tech \& M. Tech in Radio physics and Electronics, from Calcutta University (CU) in 1981, 1985 and 1987 respectively. He joined Indian Engineering Services (Group-A) in 1988 and served Department of Telecommunication, GOI. He was awarded SRF in 1992 by CSIR. Subsequently, he was awarded Postdoctoral Research Associateship in 1996 and Ph.D. in Radio physics \& Electronics from CU in 1997 with specialization in Microwave and millimeter wave systems. His research interest includes micro \& millimeter wave, semiconductor physics \& devices, Digital Image Processing, computer vision and computer animation. 
How to cite this paper: Kuldip Acharya, Dibyendu Ghoshal, " Variance Value Limited Clipping of Pentile based Sub-histogram Equalization for Contrast Enhancement of Image", International Journal of Image, Graphics and Signal Processing(IJIGSP), Vol.12, No.6, pp. 33-42, 2020.DOI: 10.5815/ijigsp.2020.06.04 